\title{
Numerical Investigation on the Response Characteristics of Hypersonic Boundary Layer under Different Types of Finite Amplitude Pulse Disturbance Waves
}

\author{
X. Tang ${ }^{1,2,3}$, J. Yu ${ }^{4 \dagger}$, H. Zhang ${ }^{2,5}, \mathrm{H} \mathrm{Li}^{2,5}$ and M. Shi ${ }^{3}$ \\ ${ }^{1}$ College of Engineering, Yantai Nanshan University, Yantai, Shandong, 265713, China \\ ${ }^{2}$ Beijing Spacecrafts, China Academy of Space Technology, Beijing 100094, China; \\ ${ }^{3}$ College of Aerospace and Civil Engineering, Harbin Engineering University, Harbin, 150001, China \\ ${ }^{4}$ China Center for Information Industry Development, Beijing 100846, China \\ ${ }^{5}$ School of Mechanical and Electrical Engineering, North China Institute of Aerospace Engineering, \\ Langfang, 065000, China
}

†Corresponding Author Email: juanyu88@ 163.com

(Received February 16, 2020; accepted July 3, 2020)

\begin{abstract}
The hypersonic transient flow pass a blunt cone under three types of pulse disturbances is calculated using DNS. The response characteristic of hypersonic boundary layer among different types of pulse disturbance is compared. The distribution and evolution characteristics of disturbance modes are investigated by mode analysis. Results indicate that the receptivity characteristics induced by freestream pulse wave have both similarities and differences with that induced by freestream continuous wave. The interactions of different types of pulse waves with boundary layer and bow shock present different characteristics. The boundary layer thermodynamic characteristics under pulse fast acoustic wave are sensitive to mainstream disturbance wave, and that under pulse slow acoustic wave are sensitive to residual reflection wave. The type of pulse disturbance wave has a great influence on the production and mode distribution of boundary layer disturbance wave. In general, the disturbance amplitude in the pulse fast acoustic wave situation is the largest, the case of entropy wave is the second, and the case of slow acoustic wave is the smallest. For regional influence, the type of pulse disturbance has a huge impact on the disturbance modes in both the head and the non-head. For the three cases of pulse wave, the main mode group attenuation phenomenon which narrows the disturbance frequency band exists in the boundary layer. This group attenuation is the fastest for freestream slow acoustic wave, followed by entropy wave, and then fast acoustic wave. Under the action of pulse slow acoustic waves, the disturbance wave evolution of each order mode in the boundary layer along the streamwise is relatively stable, followed by entropy wave, and the case of fast acoustic wave is the most active.
\end{abstract}

Keywords: Hypersonic flow; Disturbance type; Pulse wave; Mode analysis; Boundary layer.

\section{NOMENCLATURE}

$C_{f^{\prime}} \quad$ wall friction coefficient disturbance

$f \quad$ frequency

$f_{\mathrm{n}} \quad \mathrm{n}^{\text {th }}$-order harmonic frequency

F inviscid flux terms along axis $x$

$\mathbf{F}_{\mathrm{v}} \quad$ viscous flux terms along axis $x$

$\mathbf{F}^{*} \quad$ inviscid flux terms along axis $\zeta$

$\mathbf{F}_{\mathrm{v}}^{*} \quad$ viscous flux terms along axis $\zeta$

G inviscid flux terms along axis $y$

$\mathbf{G}_{\mathrm{v}} \quad$ viscous flux terms along axis $y$

$\mathbf{G}^{*} \quad$ inviscid flux terms along axis $\eta$

$\mathbf{G}_{v}^{*} \quad$ viscous flux terms along axis $\eta$

$h \quad$ characteristic length

$H_{f^{\prime}} \quad$ wall heat flux disturbance
J Jacobian matrix

$k_{\mathrm{n}} \quad$ weighted coefficient for viscous terms

$L$ positive flux term for discretization

$m_{\mathrm{n}} \quad$ weighted coefficient for positive flux terms

Maœ freestream Mach number

$n_{\mathrm{n}} \quad$ weighted coefficient for positive flux term

$O$ positive flux term for discretization

$P \quad$ pressure

$P^{\prime} \quad$ pressure disturbance

$\mathbf{Q}^{*} \quad$ state vector in the general curvilinear coordinate

Q state vector in Cartesian coordinate system

$r_{\mathrm{n}} \quad$ head radius of blunt cone

$\mathrm{Re}_{\mathrm{n}} \quad$ Reynolds number

$s \quad$ distance between the point on the wall and 


\author{
the stationary point \\ $t$ time \\ $T$ flowfield temperature \\ $T_{\mathrm{w}}$ temperature for the wall of blunt cone \\ $T_{\infty}$ freestream temperature \\ $u \quad$ velocity along axis $\mathrm{x}$ \\ $u_{\infty}$ freestream velocity \\ $u^{\prime} \quad$ velocity disturbance along axis $\mathrm{x}$ \\ $v \quad$ velocity along axis $\mathrm{y}$
}

\section{INTRODUCTION}

Since hypersonic vehicle has great advantages and potential such as fast flight speed, strong defense penetration ability and great damage power (Voland et al. 2005), (Hayashi et al. 2004) and (Venukumar et al. 2006), (Finley, 1966), the development of hypersonic vehicle has become one of the hotpots. However, there are still many technical difficulties in its development, among which the accurate prediction of aerothermodynamics characteristics of hypersonic aircraft is one of the key technical difficulties (Chen et al. 2018). Since the stability properties of hypersonic boundary layer directly determine the wall heat flux, aerodynamic force and control performance of the hypersonic aircraft (Li et al. 2010) and (Egorov et al. 2016), the design of the hypersonic aircraft is constrained. Thus, a key problem of accurate prediction of aerothermodynamics characteristics is that the stability mechanism exploration of hypersonic boundary layer has not been fully understood.

A great deal of research on the boundary layer stability mechanism in the hypersonic flow was conducted by many scholars. The transition from the laminar to turbulent in the cone boundary layer with 0 angle of attack at Mach 6 was studied by Sivasubramanian et al. (2015), and they confirmed the importance of the fundamental receptivity in the process of transition. The boundary layer transition in the hypersonic plate flow under the action of isolated roughness was also simulated by Zhao et al. (2015). This simulation showed that in the wake of the roughness unit, the instability of the shear layer is the main mechanism of the transition. With the increase of roughness, the shear layer ruptures ahead of time and the transition position tends to move forward. The effect of roughness height on supersonic boundary layer transition was studied by De Tullio (2013), and the results showed that for the roughness height close to the local displacement thickness, the growth of many unstable modes in the roughness wake is the main reason for the transition. The wind tunnel tests of Zhang et al. (2015) showed that the generation of second unstable mode is the key factor inducing transition. The strong interaction between the second and the first vorticity modes directly promotes the rapid growth of the first vorticity mode and causes to an immediate transition. Fujii (2015) further investigated the effect of two-dimensional roughness on boundary layer through hypersonic wind tunnel tests. The results showed that, in the upstream of the breakdown area, the roughness of

\author{
$v^{\prime} \quad$ velocity disturbance along axis y \\ $W \quad$ positive flux term for discretization \\ $\Delta \quad$ grid spacing \\ $\Delta t \quad$ time increment \\ $\rho_{\infty} \quad$ freestream density \\ $\rho$ density \\ $\mu_{\infty} \quad$ freestream viscous coefficient
}

wave-shaped wall with 2 delta wavelength can promote transition delaying. In addition, Shiplyuk et al. (2013) performed a detailed analysis of the laminar-turbulent transition of cones with different bluntness and freestream waves, and observed that the instability of second-order mode is the main cause of laminar-turbulence transition and with the increase of bluntness, the motion range of laminar flow gradually enlarges. Among these investigations, it wss found that receptivity process induced by external disturbance has a great effect on the stability analysis of the whole boundary layer. In view of the fact, the boundary layer receptivity in hypersonic flow to $2 \mathrm{D}$ and $3 \mathrm{D}$ wall disturbances was numerical investigated by Wang et al. (2006). These simulations indicated that the boundary layer receptivity to $2 \mathrm{D}$ wall disturbances is independent of the specific type and distribution of disturbances. It was also showed that boundary layer flow is more susceptible to blowing and suction than temperature disturbance and wall vibration. Wang et al. (2018), using high-order finite difference scheme, further investigated the roughness effects in the hypersonic boundary layer receptivity process induced by pulse entropy disturbance, and pointed out that the different disturbance are magnified in the upper part of the roughness element and repressed in the lower part. However, with the increase of boundary layer disturbance frequency, the influence of roughness is gradually weakened. The numerical investigation of the nose bluntness influence on the receptivity of boundary layer caused by freestream acoustic was performed by Zhong et al. (2011), and they showed that dimensionless frequencies can approximate wave structures with different bluntness and frequencies. The same instability threshold of second mode can result in an instability location in downstream under different nose bluntness. The investigation on the boundary layer receptivity due to different amplitude acoustic disturbances conducted by Tang et al. (2017) found a positive correlation between the maximum amplitude of boundary layer disturbance mode and the disturbance amplitude in freestream. Zhao et al. (2018) investigated, under local heat or cool source, the boundary layer disturbance evolution in a hypersonic flat-plate at Mach 6, and pointed out that the heat or cool source position has an important impact on Mode S. Besides this, the boundary layer receptivity study of Wang et al. (2018) found that for the slow acoustic waves with low-frequency, the slow acoustic path propagating downstream of shock wave acts a dominant role. Near the neutral 
point, the entropy layer unstable disturbance wave can promote the first mode with lower receptivity efficiency. Throughout these studies, it can be found that in the receptivity study of hypersonic boundary layer, the interaction of external disturbance with shock waves and hypersonic boundary layer as well as the mechanism of the production and evolution of boundary layer disturbance wave were mainly investigated by introducing disturbance sources in hypersonic flowfield. According to the selection of disturbance sources, they can be divided into freestream disturbances (such as fast acoustic disturbance, slow acoustic disturbance, entropy disturbance, vorticity disturbance, etc.) and wall source disturbances (such as rough element, wall vibration, wall blowing and suction, wall thermal state, etc.) in the studies of hypersonic boundary layer receptivity. In the past few decades, many scholars have done a lot of work on the receptivity characteristics of hypersonic boundary layer induced by freestream disturbance. The direct numerical simulations of Egorov et al. (2008) and Duan et al. (2010) investigated the Mach number effects on the boundary layer of compressible turbulent and acoustic disturbance, and revealed the new characteristics of the front disturbance field of the boundary layer plate. Qin et al. (2016) further analyzed the boundary layer receptivity in hypersonic flow to different freestream disturbance. They pointed out that the disturbances with any of the three basic types of frequency and wavelength interacts with the shock wave, resulting in acoustic disturbances reflected between the wall and the shock. The numerical investigation of Wang et al. (2014) confirmed that, along the streamwise, the frequency band narrows and the number of main disturbance group decreases. The nose bluntness effect studies of Huang et al. (2014) and Zhong et al. (2011) showed that the interfere between freestream hotspot and shock wave produce fast acoustic waves that dominate the receptivity process, and also confirmed the bluntness effect on the instability location in the downstream. Gao et al. (2015) also pointed out that the nonlinear interaction caused by slow acoustic disturbances and fast acoustic disturbances stimulate the second mode by generating sum-frequency interference. The studies mentioned above show that disturbance wave types and parameters have significant effect on the interfering of disturbance with hypersonic flow, the disturbance wave evolution, as well as the boundary layer receptivity. However, these studies are mainly based on continuous small disturbances. The response characteristics of hypersonic boundary layer to continuous small disturbances in the freestream, the linear and non-linear evolution mechanism of disturbance waves along the streamwise under freestream disturbance waves are mainly focused. As a distinct disturbance form different from continuous disturbance, the receptivity characteristics of boundary layer caused by finite amplitude pulse wave disturbance in the freestream are subtly different from that caused by continuous small disturbance in the freestream. Therefore, in the context that the hypersonic boundary layer stability mechanism has not been fully understood, there are reasons to believe that the investigation on the hypersonic boundary layer receptivity induced by different types of freestream finite amplitude pulse waves can provide a new unique perspective for understanding the receptivity mechanism and even the boundary layer stability mechanism. However, a systematic research in this field has not been attempted.

Therefore, based on a blunt cone with $8^{\circ}$ half-cone angle at Mach 6, DNS of hypersonic flowfield with different types of finite amplitude pulse disturbances in freestream is performed in this paper. The effects of finite amplitude pulse disturbance on flowfield and boundary layer are discussed, and the boundary layer receptivity characteristics caused by different types of finite amplitude pulse disturbances are studied.

\section{GoVERNING EQUATION NUMERICAL METHOD}

\subsection{Governing Equation}

To facilitate the solution, the $\mathrm{N}-\mathrm{S}$ equation in Cartesian coordinate $(x, y)$ is transformed into curvilinear coordinate $(\zeta, \eta)$ by coordinate transform method. The two dimensional N-S equation in curvilinear coordinate can be expressed as Eq.(1):

$\frac{\partial \mathbf{Q}^{*}}{\partial t}+\frac{\partial\left(\mathbf{F}^{*}-\mathbf{F}_{v}^{*}\right)}{\partial \xi}+\frac{\partial\left(\mathbf{G}^{*}-\mathbf{G}_{v}^{*}\right)}{\partial \eta}=0$

Here $\mathbf{Q}^{*}, \mathbf{F}^{*}, \mathbf{G}^{*}, \mathbf{F}_{\mathrm{v}}^{*}$ and $\mathbf{G}_{\mathrm{v}}^{*}$ are expressed as Eq.(2):

$\begin{cases}\mathbf{Q}^{*}=\frac{\mathbf{Q}}{\mathbf{J}} & \mathbf{F}^{*}=\frac{\mathbf{F}}{\mathbf{J}} \\ \mathbf{F}_{\mathrm{v}}^{*}=\frac{\mathbf{F}}{\mathbf{J}} & \mathbf{G}_{\mathrm{v}}^{*}=\frac{\mathbf{G}}{\mathbf{J}}\end{cases}$

where $\mathbf{J}$ can be expressed as Eq.(3):

$\mathbf{J}=\left|\begin{array}{ll}\xi_{x} & \xi_{y} \\ \eta_{x} & \eta_{y}\end{array}\right|$

\subsection{Numerical Method}

In present work, the hypersonic blunt cone flow with pulse disturbance wave is simulated. The discontinuous characteristics will occur in the hypersonic transient flowfield induced by the pulse disturbance wave, and numerical oscillation and dissipation will easily occur in the numerical calculation of the discontinuous area. In view of this, excellent numerical methods should be used to suppress numerical dissipation and numerical oscillation.

The numerical method of total variation decreasing (TVD) scheme can effectively suppress the numerical dissipation phenomenon and ensure the convergence of the calculation results. The thirdorder TVD Runge-Kutta scheme is widely used in the time marching of high-order unsteady flowfield, and achieves good results (Kara et al. 2011). Therefore, the third-order TVD Runge-Kutta scheme is applied to discretize the time term in 


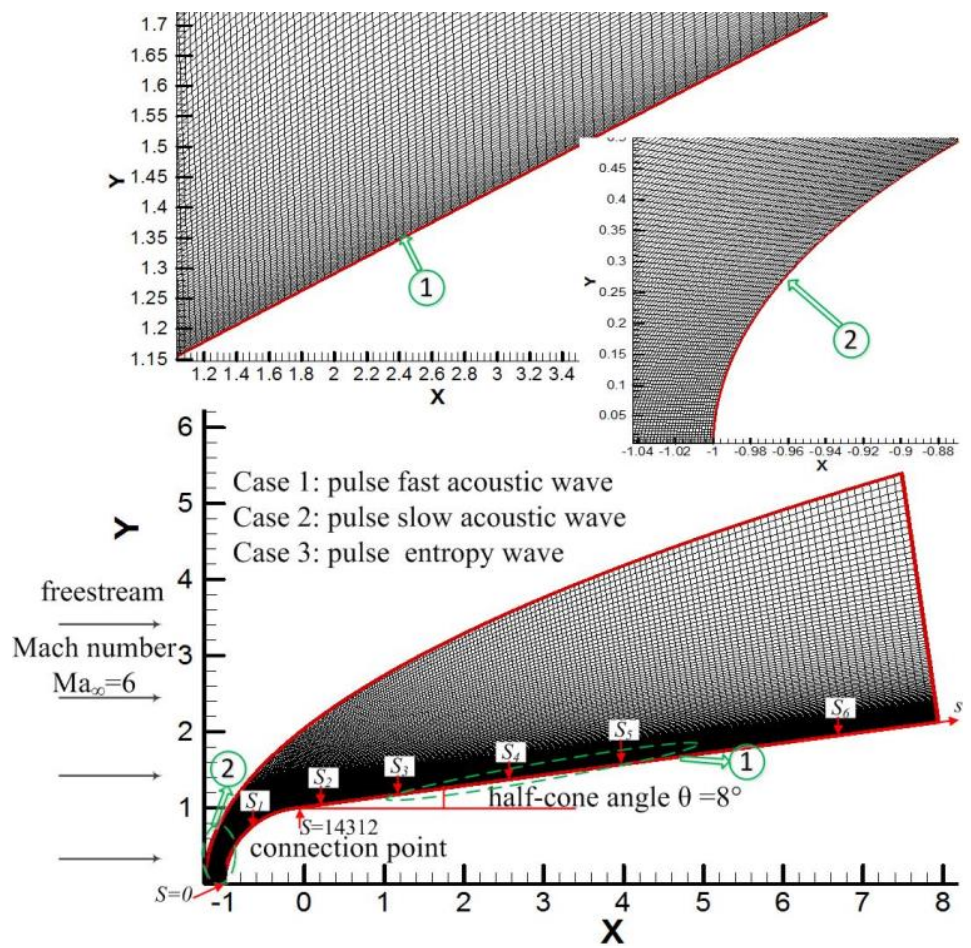

Fig. 1. Computing model and mesh.

present work. For numerical schemes without TVD properties, some terms of the equation can be multiplied by a reasonable non-linear function, so that these difference equations satisfy TVD conditions. However, this will affect the accuracy of discrete schemes and reduce the resolution of discontinuous solutions. Because of this, the Weighted Essential Non-Oscillatory (WENO) schemes and the improved WENO schemes are no longer strict with TVD properties through the innovation of computational methods. At the same time, WENO schemes can effectively suppress numerical oscillations in discontinuous areas, thus maintaining high computational accuracy for the calculation results. Especially for the discretization of the inviscid flux term, it can maintain good performance (Liu et al. 1994). Therefore, WENO schemes and improved WENO schemes are also widely applied in the DNS of complex compressible flowfields. At the same time, considering that the central difference scheme (CDS) can achieve good results (Li et al. 2008) in the viscous terms discretization, the sixth-order CDS is applied to discretize viscous terms in this paper.

Therefore, the N-S equation of compressible flow in general curvilinear coordinates is solved by highorder precision discretization method in present work. The inviscid terms in the $\mathrm{N}-\mathrm{S}$ equation are decomposed into positive flux terms and negative flux terms by $\mathrm{S}-\mathrm{W}$ method, which are discretized by fifth-order upwind WENO schemes. The discrete format for the positive flux terms is as:

$W^{\prime}=\frac{m_{1} W_{j+3}+m_{2} W_{j+2}+m_{3} W_{j+1}+m_{4} W_{j}+m_{5} W_{j-1}+m_{6} W_{j-2}}{\Delta}$
The discrete format for the negative flux terms is as:

$O^{\prime}=\frac{n_{1} O_{j+4}+n_{2} O_{j+3}+n_{3} O_{j+2}+n_{4} O_{j+1}+n_{5} O_{j}+n_{6} O_{j-1}}{\Delta}$

The discrete format for viscous terms is as:

$L^{\prime}=\frac{k_{1}\left(L_{j+1}-L_{j-1}\right)+k_{2}\left(L_{j+2}-L_{j-2}\right)+k_{3}\left(L_{j+3}-L_{j-3}\right)}{\Delta}$

The discrete format for the time term is as:

$\left\{\begin{array}{l}U^{(1)}=U^{n}+\Delta t L\left(U^{(n)}\right) \\ U^{(2)}=\frac{3}{4} U^{(1)}+\frac{1}{4}\left[U^{(1)}+\Delta t L\left(U^{(1)}\right)\right] \\ U^{n+1}=\frac{1}{3} U^{n}+\frac{2}{3}\left[U^{(2)}+\Delta t L\left(U^{(2)}\right)\right]\end{array}\right.$

In the formula, $\Delta t=5 \times 10^{-4}$.

\section{Computational Conditions AND MODEL}

Since blunt cone is an aerodynamic configuration existing extensively in hypersonic vehicle, it was chosen as the computing model in many studies on the receptivity characteristics of hypersonic boundary layer (Zhang et al. 2008; Prakash et al. 2011; Wan et al. 2018). So, a blunt cone with $8^{\circ}$ half-cone angle and 6 Mach freestream is introduced in this paper. Meanwhile, in order to reduce computational complexity, a 2D model with head radius $r_{n}=1 \mathrm{~mm}$ is selected. To avoid the angleof-attack (AOA) effects on the response characteristics of boundary layer, a 0 degree angleof-attack freestream is selected in present computation. Figure 1 shows the computing model 
and mesh. The freestream temperature $T_{\infty}=169 \mathrm{~K}$. The freestream Mach number $M_{\infty}=6$. The freestream Reynolds number $R e_{\mathrm{n}}=\left(\rho_{\infty} r_{\mathrm{n}} u_{\infty}\right) / \mu_{\infty}=6000$. The isothermal wall temperature $T_{\mathrm{w}}=200 \mathrm{~K}$.

In present simulation, freestream condition is used at the upstream boundary, and export extrapolation condition is applied at the outflow boundary. At the wall boundary of blunt cone, no-slip condition and no-penetration are used. Dimensionless parameterization is carried out for the flowfield variables, that is $T / T_{\infty}, P / \rho_{\infty} u_{\infty}, t /\left(r_{\mathrm{n}} / u_{\infty}\right), v / u_{\infty}, u / u_{\infty}$, $h / r_{\mathrm{n}}$. Superscript "'” represent the disturbance of flowfield variables, and the disturbance of flowfield variables are expressed as the transient flowfield parameters with respect to the base flowfield parameters (Zhang et al. 2008).

The simulation conducted in present work is resolved by $300 \times 120$ grids. The variable spacing mesh is employed to the streamwise and the normal of blunt cone wall. The grid nodes in the areas near the wall and blunt cone head are appropriately increased. To make it clearer, the grid details of boundary layer are magnified in Fig.1. The mesh size in present work is commensurate with that in similar models of hypersonic flowfield simulations (Zhang et al. 2008; Prakash et al. 2011).

A body-fitted coordinate $\mathrm{s}$ is introduced in present work, which indicates the distance between the point on the wall and the stationary point on the blunt cone. The relation between $s$ and $x$ in the Cartesian coordinate is as follows.

$x= \begin{cases}-\cos (s) & s \leq \frac{\pi}{2}-\theta \\ \left(s-\frac{\pi}{2}+\theta\right) \cos (\theta)-\sin (\theta) & s>\frac{\pi}{2}-\theta\end{cases}$

In present work, three cases of the hypersonic unsteady flow pass a blunt cone induced by freestream disturbances (Case 1: pulse fast acoustic wave, Case 2: pulse slow acoustic wave, Case 3: pulse entropy wave) are investigated. The expressions of pulse slow acoustic disturbance, pulse fast acoustic disturbance and pulse entropy disturbance are shown as Eq. (9), Eq. (10) and Eq. (11), respectively.

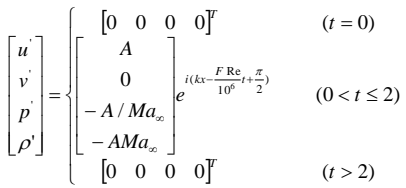

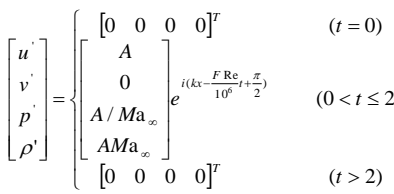

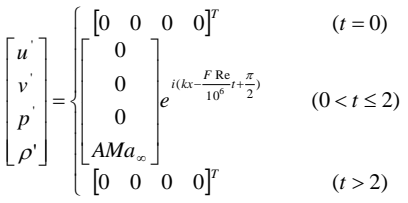

Among them, $A=4 \times 10^{-2}, \mathrm{Ma}_{\infty}=6$, the generalized frequency $F=50 \pi$ (corresponding to $f=0.25$ ), and the disturbance variable $k=3.144 \times 10^{-4}$.

According to the expressions of different types of disturbance waves and the state equation of the ideal gas, the distinction between the types of disturbance waves can be obtained. Namely, for slow acoustic disturbance, the max disturbance amplitudes of $u^{\prime}, v^{\prime}, P^{\prime}, \rho^{\prime}$ and $T^{\prime}$ are equal to $A, 0$, $-A / M a_{\infty}, \quad-A \times M a_{\infty}$ and $\left(-A / M a_{\infty}+A / M a_{\infty}\right) /(1-$ $\left.A / M a_{\infty}\right)$, respectively; for fast acoustic disturbance, the max disturbance amplitudes of $u^{\prime}, v^{\prime}, P^{\prime}, \rho^{\prime}$ and $T^{\prime}$ are equal to A, $0, A / M a_{\infty}, A \times M a_{\infty}$ and $\left(A / M a_{\infty}-\right.$ $\left.A / M a_{\infty}\right) /\left(1+A / M a_{\infty}\right)$, respectively; for fast entropy disturbance, the max disturbance amplitudes of $u^{\prime}$, $v^{\prime}, P^{\prime}, \rho^{\prime}$ and $T^{\prime}$ are equal to $0,0,0, A \times M a_{\infty}$ and ($\left.A / M a_{\infty}\right) /\left(1+A \times M a_{\infty}\right)$, respectively. The steady hypersonic flowfield is first calculated $(t=0)$ by transient simulation, and then three kinds of freestream pulse disturbance waves (fast, slow and entropy waves) with half period ( $t=2)$ are separately added to the flowfield at the upstream boundary.

\section{Results And Discussion}

\subsection{Verification of Grid Independence and Numerical Method}

To test the numerical scheme reliability, the numerical simulation verification is conducted in our previous works (Tang et al. 2014). Due to there is no available data about the present model, a similar hypersonic flowfield (a blunt cone with $0^{\circ}$ AOA and $0^{\circ}$ half-cone angle, Mach=7.1) is numerical calculated. Figure 2 shows the comparison of shock standoff distance between present work and literatures (Lobb, 1964; Prakash et al. 2011). The calculation result in this paper is in accordance with the results from Lobb's experiment and Prakash et al's simulation.

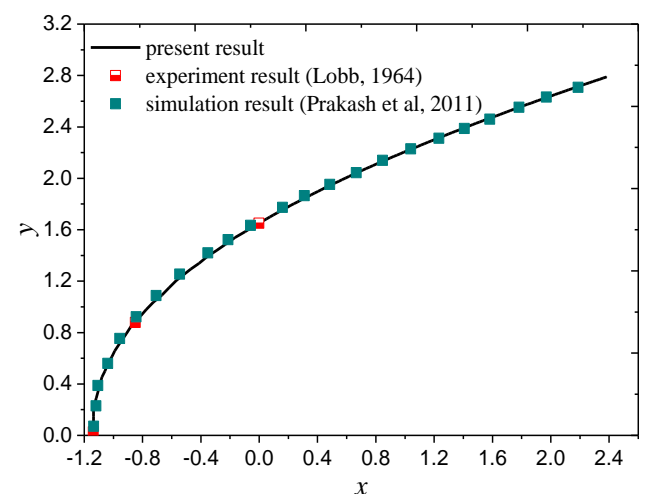

Fig. 2. Comparison of shock distance between present work and literatures (Lobb, 1964; Prakash et al. 2011, Tang et al. 2014).

To verify the validity of grid independence in present work, the numerical simulations with $300 \times 120$ grids and $450 \times 180$ grids are conducted respectively. Figure 3 shows the comparison of the friction coefficient disturbance between $300 \times 120$ grids and $450 \times 180$ grids. The calculation result proves the validity of grid independence. 


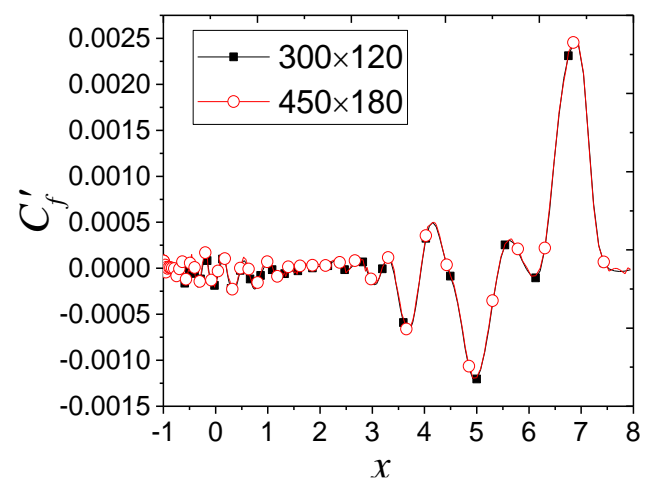

Fig. 3. Comparison of the friction coefficient disturbance between $300 \times 120$ grids and $450 \times 180$ grids.

\subsection{Response Characteristics of Hypersonic Flowfield}

Figure 4 shows the velocity disturbance contour of hypersonic flowfield under different freestream pulse waves. It can be seen, for all the three cases, the velocity of the flowfield near the wall and the shock wave varies significantly. However, it can also be seen from the figure that in the region where the shock wave interacts with the freestream pulse disturbance, the velocity of the flowfield increases under Case 1, decreases under Case 2, and increases under Case 3. But the variation magnitude of the velocity under Case 3 is smaller than that under Case 1 and Case 2. From Fig.4, it is obtained that, the velocity disturbance pattern near the wall change significantly for all the three cases. The remarkable change of the velocity disturbance near the wall indicates that the pulse disturbance wave in the freestream has a great effect on the strong shear flow structure of the boundary layer. There are also significant differences in velocity disturbance patterns near the wall among the three cases. Under Case 1, there is only an elliptical area near the boundary layer of $5.0<x<6.0$ with a significant increase in velocity. Compared with the amplitude of velocity variation in this area, the velocity variation in other areas of the boundary layer is small. This shows that the mainstream disturbance wave has a significantly stronger effect on the boundary layer than the reflected wave in Case1. Under Case 2, there is also an area near the boundary layer of $5.0<x<6.0$ where the velocity increases significantly due to mainstream disturbance wave, but the velocity of the flowfield near the wall of $3.0<x<5.0$ increases or decreases obviously. It is known, the change of the velocity of the flowfield near the wall of $3.0<x<5.0$ is caused by the reflection wave. This indicates that the reflection wave has a stronger effect on the boundary layer compared to the mainstream disturbance wave in the case of slow acoustic wave. Under Case 3, similar to Case 2, there is an area near the boundary layer of $5.0<x<6.0$, where the velocity of the flowfield increases significantly; meanwhile, the velocity of the flowfield near the boundary layer of $3.0<x<5.0$ increases or decreases obviously. It is obtained that, besides the relatively strong influence of the mainstream disturbance wave on the boundary layer flow, the reflected wave is also a factor that cannot be ignored under the condition of Case 3 . The difference of the velocity disturbance within the three cases makes the boundary layer vortices develop discrepantly, which may lead to different mechanisms of vortex breakdown and the boundary layer stability characteristics.
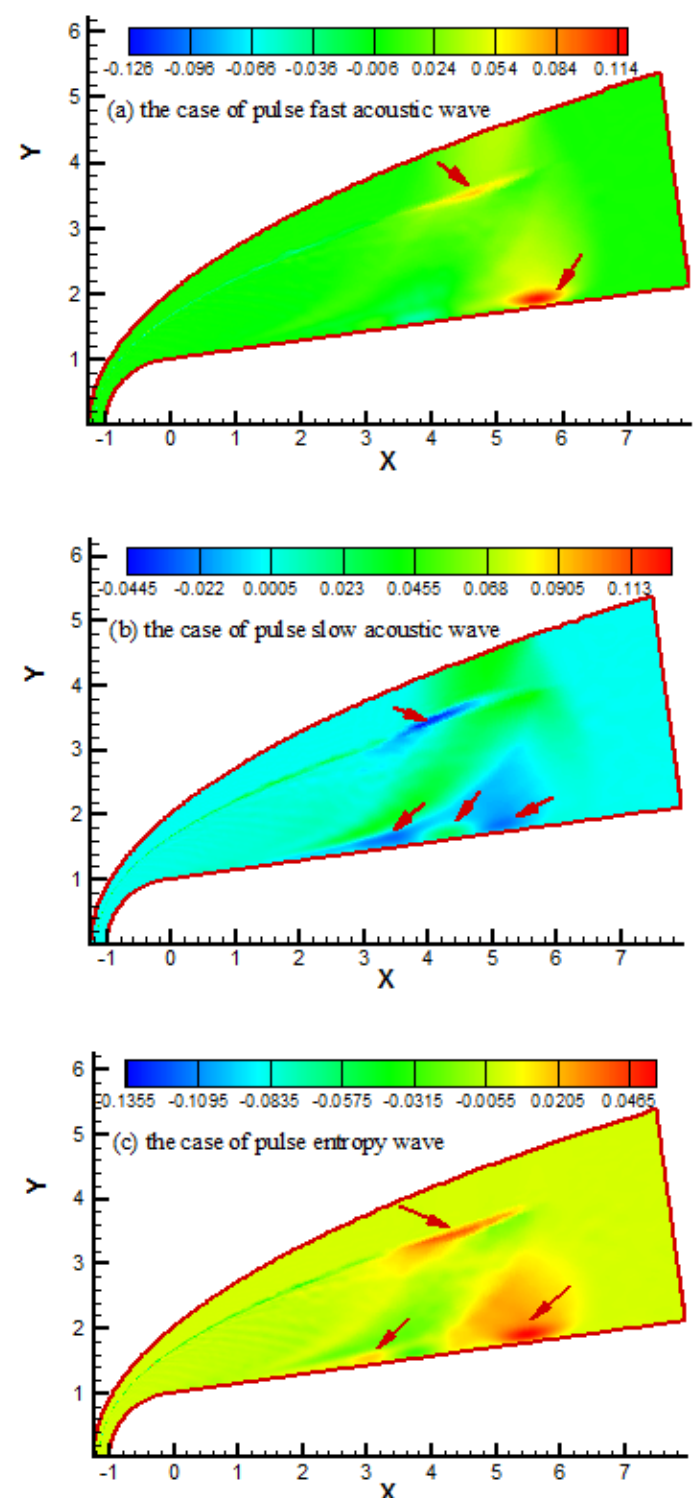

Fig. 4. Velocity disturbance contour under different freestream disturbance waves. (a) Velocity disturbance under pulse fast acoustic wave. (b) Velocity disturbance under pulse slow acoustic wave. (c) Velocity disturbance under pulse entropy wave.

Figure 5 shows the comparison of disturbance isogram of velocity along axis $x\left(u^{\prime}\right)$ and axis y $\left(v^{\prime}\right)$ between pulse fast acoustic wave and continuous fast acoustic wave. The number in Fig. 5 is the level of velocity disturbance, as is shown in the 
legend of the Figure. From the figure, it is obtained that whether under the condition of pulse wave or continuous wave, the areas with violent change of velocity disturbance are mainly concentrated near the boundary layer and the shock wave. Under the view of the velocity disturbance strength in different areas, the velocity disturbance strength in the head is significantly greater than that in the non-head in the condition of freestream continuous wave, while in the condition of freestream pulse wave, the velocity disturbance strength in the areas near the mainstream disturbance wave and the initial reflected wave is significantly greater than that in the other areas. The velocity disturbance patterns due to continuous wave are obviously distinct from those due to pulse wave. In the condition of continuous wave, the velocity disturbance patterns present obvious periodicity, while the velocity disturbance patterns have no obvious periodicity in the case of pulse wave.

Since wall shear force is directly determined by wall friction coefficient $\left(C_{f}\right)$, and $C_{f}$ is an important indicator for characterizing the boundary layer shear structure, the key to studying the boundary layer stability is to reveal the stability characteristics of the boundary layer shear structure. Therefore, the response characteristics of the wall friction coefficient will be analyzed below. Figure 6 shows the wall friction coefficient disturbance distributions under three types of pulse disturbances at different times. In Fig.6 and the following sections, "fw", "sw" and "ew" are used to represent Case 1, Case2 and Case3 respectively. As can be seen from Fig. 6(a), at $t=1.0$, the wall friction coefficient increases under Case1 and Case3, however, under Case2, the wall friction coefficient decreases. Obviously, $C_{f}$ is mainly affected by the mainstream disturbance wave at $t=1.0$, and the effect of mainstream disturbance on the $C_{f}$ in Case 2 is the reverse of that in Case1 and Case 3 . At the same time, the disturbance amplitude of $C_{f}$ in Case 1 is different from that in Case3. In general, the disturbance amplitude of $C_{f}$ in Case 1 is the largest, followed by Case 3, and that in Case 2 is the smallest. It can also be seen that within the three cases, the disturbance distribution of the $C_{f}$ under the action of reflected wave is also notably different; meanwhile the variation trends of $C_{f}^{\prime}$ approximately in the area of $3<x<5$ for all the three cases are similar, as the circle area shown in Fig.6(d). Compared with Case1 and Case3, the reflection wave due to freestream pulse slow acoustic wave has the greatest influence on $C_{f}$. The influences of the three types of pulse disturbances on wall friction coefficient are significantly different, which indicates that the action of different pulse waves with the boundary layer shear structure have different mechanisms. It is concluded that the boundary layer shear structures under the three types of pulse disturbances represent different stability properties.

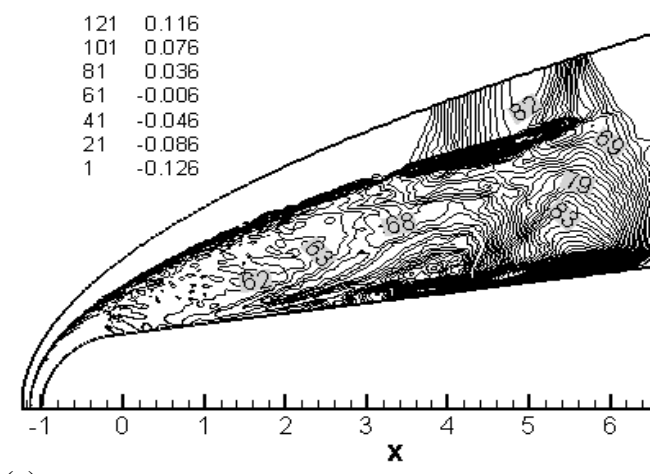

(a)

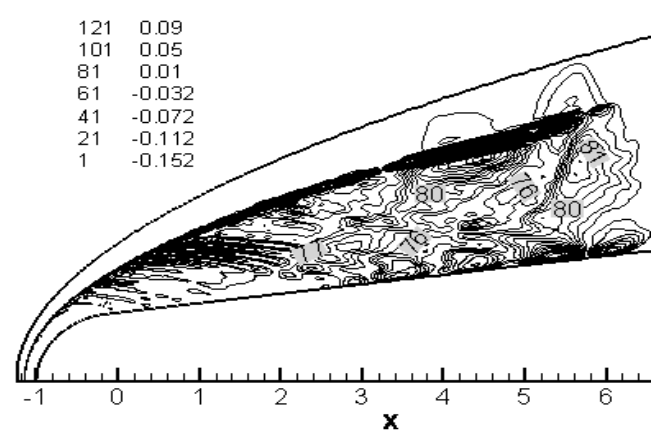

(b)

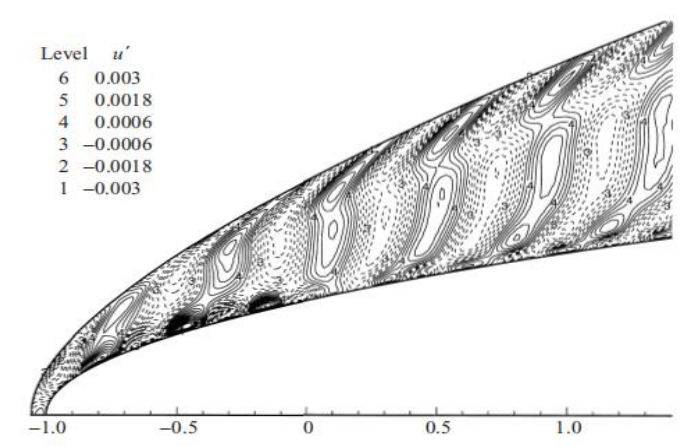

(c)

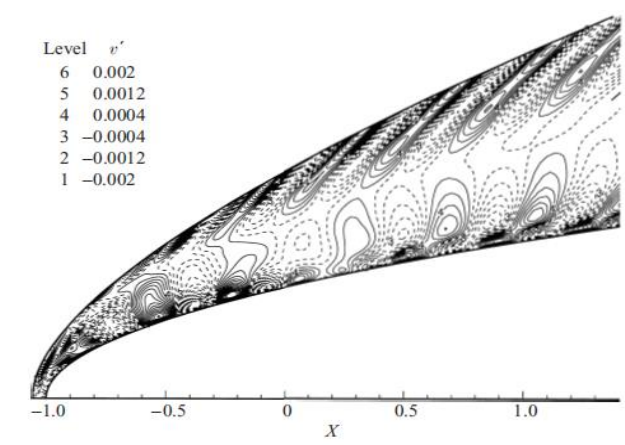

(d)

Fig. 5. Comparison of disturbance isogram of velocity along axis $x\left(u^{\prime}\right)$ and axis $y\left(v^{\prime}\right)$ between pulse fast acoustic wave and continuous fast acoustic wave. (a) $u^{\prime}$ for pulse fast acoustic wave.

(b) $v$ 'for pulse fast acoustic wave. (c) $u^{\prime}$ for continuous fast acoustic wave(Zhong 2001). (d) $v^{\prime}$ for continuous fast acoustic wave (Zhong 2001). 

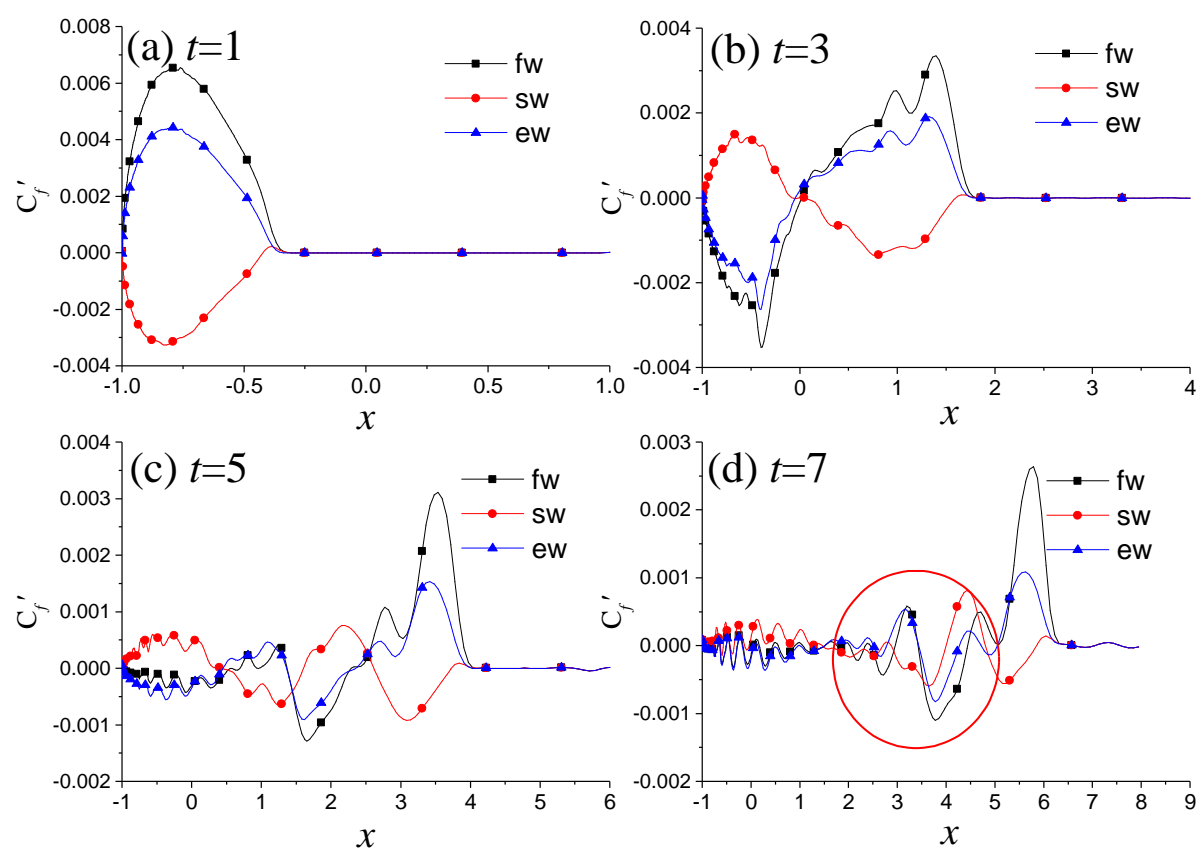

Fig. 6. Comparison of the distribution of wall friction coefficient disturbances under different freestream pulse waves at $t=1(\mathrm{a}), t=2(\mathrm{~b}), t=5(\mathrm{c})$ and $t=7(\mathrm{~d})$.

Similarly, heat flux is another key parameter for characterizing the thermodynamic characteristics. In order to describe the effects of different pulse waves on the boundary layer thermal state, and to analyze the differences of their effects on the thermodynamic characteristics, Figure 7 shows the comparison of wall heat flux disturbances $\left(H_{f}^{\prime}\right)$ at $t=8.0$ under the three cases. It is obtained that in the area of $6<x<8$, the wall heat flux disturbance under Case1 is the largest, followed by Case3, and the minimum is Case2. Obviously, this is the result of the action of the mainstream disturbance wave, which indicates that the influence of the mainstream disturbance produced by freestream pulse entropy wave and slow acoustic wave on the boundary layer thermodynamic characteristics is less than that produced by freestream pulse fast acoustic wave. However, in the area of $-1<x<6$, wall heat flux disturbance under Case 2 is obviously larger than that under the other two cases; obviously that is caused by the residual reflection wave. In other words, the residual reflection wave produced by the freestream pulse slow acoustic wave has a larger impact on the boundary layer thermodynamic characteristics than the other two cases. It is obtained that the distribution of $H_{f}^{\prime}$ on the wall of hypersonic blunt cone is significantly different under the three types of pulse disturbances. The wall thermal state directly changes the boundary layer thermal state, and the boundary layer shear structure will be significantly affected by its thermal state. Moreover, the change of the boundary layer thermal state affects the wall thermal state in turn. Thus, the boundary layer thermal state and the wall thermal state will interfere with each other, which alter the boundary layer stability characteristics. From Fig.6 and Fig.7, it is concluded that the stability mechanism of boundary layer is affected by different types of freestream pulse disturbances. Consequently, the boundary layer stability characteristics under the three cases will be discussed below.

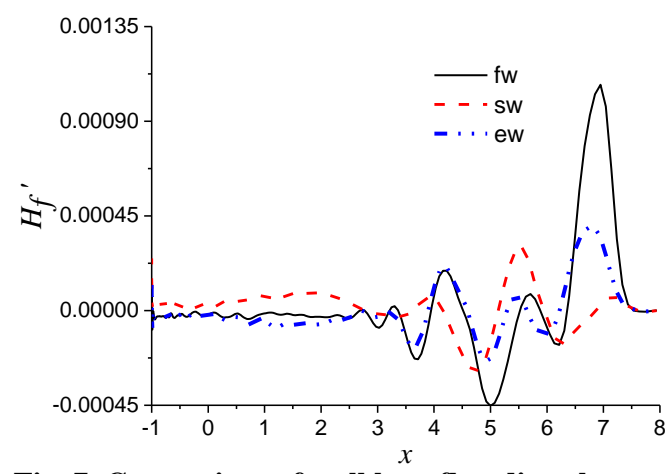

Fig. 7. Comparison of wall heat flux disturbances at $t=8.0$ under different types of pulse disturbance waves.

To discuss the evolution trend of boundary layer disturbance along the streamwise at different position points on the wall of blunt cone $(s=1.15828,1.77468,2.80255,4.11777,5.63955$ and 8.39659) is selected to record the variation of boundary layer disturbance under different freestream pulse waves. The time-domain signal of boundary layer pressure disturbance $P^{\prime}(x, y, t)$ at different locations under different freestream pulse waves is shown in Figure 8. Figure 8 shows that the time-domain variation trends of boundary layer pressure disturbance are different under the three cases. Under Case2, the boundary layer pressure disturbance first decreases, while the boundary layer pressure disturbance first increases under 

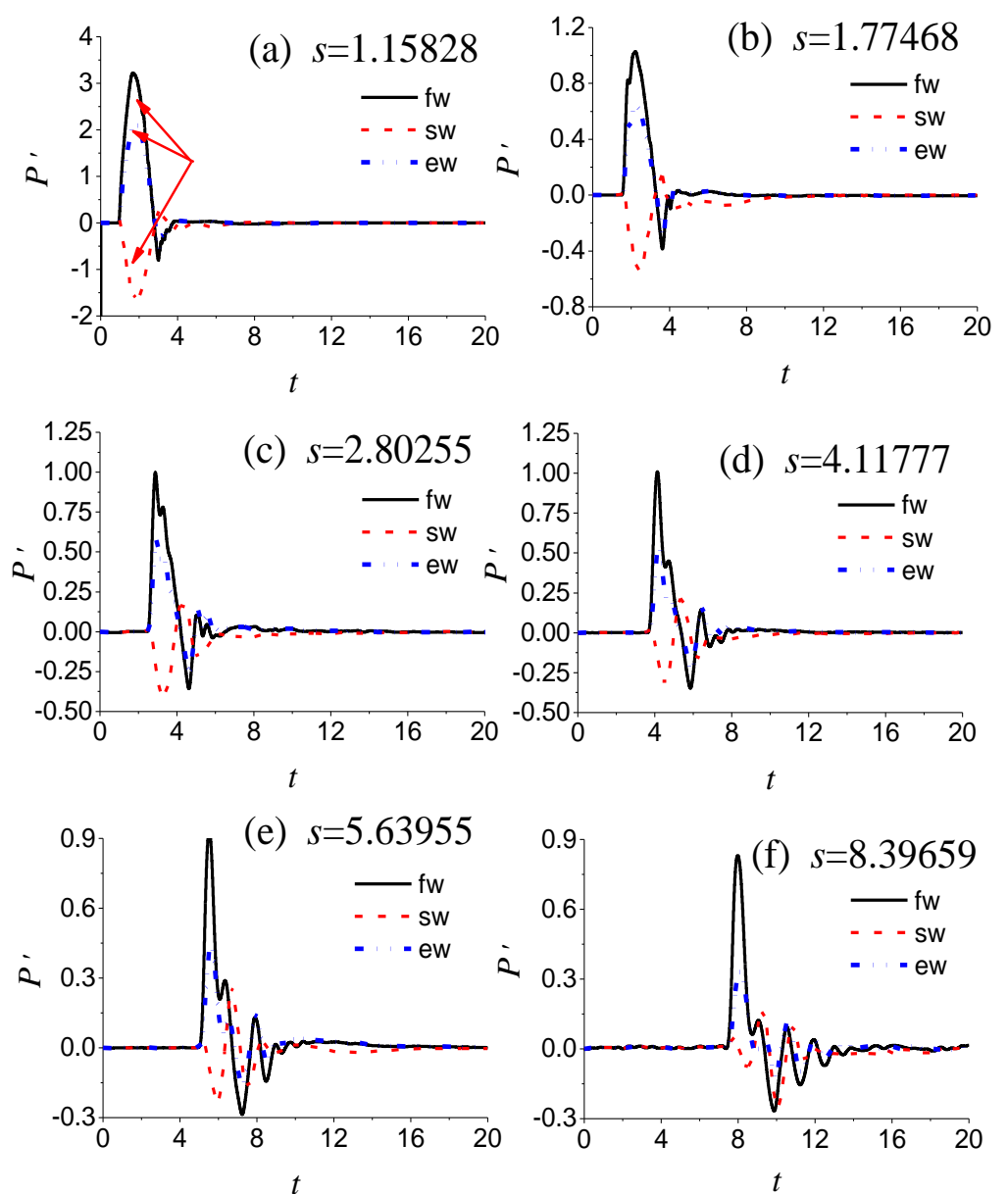

Fig. 8. Time-domain signal of boundary layer pressure disturbance $\left.P)^{\prime} x, y, t\right)$ at $s=1.15828(\mathrm{a})$, $1.77468(b), 2.80255(c), 4.11777(d), 5.63955(e)$ and 8.39659(f).

Case 1 and Case 3, as indicated by the arrow in Fig.8. Whether in the downstream (non-head area) or in the upstream (head area), the amplitude of boundary layer pressure disturbance under the case of pulse fast acoustic wave is the largest. However, in the three cases, the boundary layer pressure disturbance signal has a similar trend, that is, it is a damped vibration process until the vibration magnitude drops to zero. Among the three cases, the pressure disturbance amplitude in the head area $(s=1.15828)$ is much larger than that the non-head area $(s=2.80255,4.11777,5.63955,8.39659)$. This is because the bow shock enlarges the freestream disturbance wave. The bow shock near the head is a normal shock and an oblique shock behind the head. The magnifying effect of the normal shock to boundary layer pressure disturbance is stronger than that of the oblique shock.

\subsection{Mode Analysis of Boundary Layer}

Figure 9 shows the mode analysis of pressure disturbance under different pulse waves at different positions. From Fig.9, the type of pulse disturbance wave has a great influence on the production, distribution and mode evolution of boundary layer disturbance wave. In general, the Fourier transform amplitude (FTA) of boundary layer disturbance in
Case1 is the largest, that induced by pulse entropy wave (Case3) is the second, and that induced by pulse slow acoustic wave (Case2) is the smallest. However, when $s>1.77468$, the FTA of pressure disturbance near the frequency band of $0.5<f<0.75$ under Case 2 is larger than that under Case1 and Case3. It can also be seen that when $s=1.15828$, the trend of Fourier transform amplitude-frequency curve of boundary layer pressure disturbance under the three cases is similar, that is, the disturbance modes with maximum amplitude distribute in the range of $f<0.5$, and there are four obvious peaks or four main mode groups. The four main mode groups are distributed near the frequencies $f=0.25$, $1.0,1.5$ and 2.0 , that is, near $f=0.25 \mathrm{n}$ ( $\mathrm{n}$ is an integer). As the boundary layer disturbance wave evolves from the upstream to the downstream, when $s=1.77468$, the FTA of boundary layer pressure disturbance decreases significantly in all the three cases. The FTA at $s=1.77468$ is several times smaller than at $s=1.15828$ (the head area), while the FTA-frequency curves of pressure disturbance under different cases remain similar. At $s=2.80255$, there are still four distinct peaks in the FTAfrequency curve under Case 1 and Case 3 , but the four peaks are no longer distributed near $f=0.25 \mathrm{n}$ (n is an integer), which indicates that the boundary 

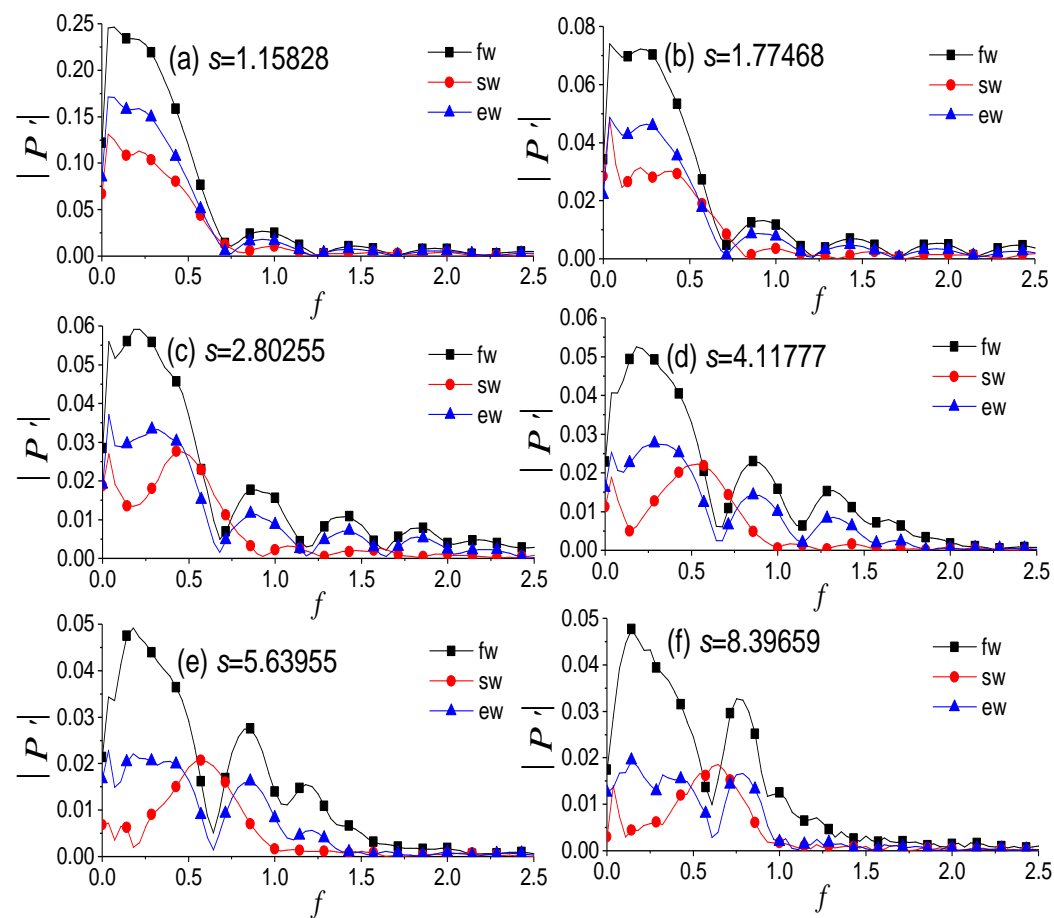

Fig. 9. Mode analysis of pressure disturbance under different pulse waves at $s=1.15828(a), 1.77468(b)$, 2.80255(c), 4.11777(d), 5.63955(e) and 8.39659(f).

layer main mode groups exist migration phenomenon. The figure also shows that when $s=2.80255$, only one of the main mode group distributed near $f=0.5$ exists in the boundary layer in Case2, while the other main mode groups attenuate significantly. When $s>4.11777$, the number of main mode group under Case 1 and Case 3 also begins to decrease, and the pace of decline in Case 3 is faster than that in Case1. Therefore, it can be concluded that the main mode group attenuation phenomenon which narrows the disturbance frequency band exists in all of the three cases, and the fastest attenuation occurs in Case2, followed by Case 3 and the slowest in Case1.

In the study of (Zhang et al. 2008), the response of a blunt body boundary layer to continuous disturbance waves in the hypersonic freestream was studied, and the differences of receptivity characteristics among different types of continuous disturbance waves were analyzed. Given that there are both some similarity and significant difference between freestream pulse waves and freestream continuous waves. A contrastive analysis of the boundary layer response to continuous disturbance waves and pulse disturbance waves is employed in present work. Figure 10 shows the evolution of Fourier transforms module values (FTMV) of boundary layer pressure disturbance along the streamwise for freestream pulse waves and continuous waves (Zhang et al. 2008). The Fourier transforms amplitude (FTA) of pressure disturbance in Case 1 is the largest, that in Case 3 is the second, and that in Case 2 is the smallest. As shown in Fig.10, whether under the case of freestream pulse wave or continuous wave, the FTMV of the pressure disturbance caused by fast acoustic wave is the largest, that caused by entropy wave is the second, and that caused by slow acoustic wave is the smallest. In general, the FTMV in the boundary layer decreases along the streamwise, and no matter in the case of continuous wave or pulse wave, all of the FTMV curves along the streamwise present a turning point near the connection area between the head and the non-head. However, the evolution laws of FTMV of boundary layer pressure disturbance along the streamwise are found to be significantly different between the conditions of freestream pulse wave and continuous wave. After the turning point of the FTMV attenuation rate near the connection area between head and non-head, the FTMV attenuation rate in the case of pulse wave rapidly becomes small, while that in the case of continuous wave slightly decreases. With the evolution from the connection area to the non-head area, the FTMV of pressure disturbance decreases continuously and slowly in the case of pulse wave, while in the condition of continuous wave, the FTMV of disturbance decreases rapidly at first, then increases slightly, and then continues to decay slowly after another turning point. It is revealed that there are both some similarity and significant difference between the boundary layer receptivity characteristics due to pulse wave and those due to the continuous wave.

From Fig.9 and Fig.10, it can be obtained that different disturbance modes along the streamwise under different freestream waves present different variation trends and distribution characteristics. To analyze the evolution of different boundary layer modes along the streamwise and the influences of freestream pulse type, the development of the fundamental mode $\left(f_{1}=0.25\right)$ and harmonic modes 

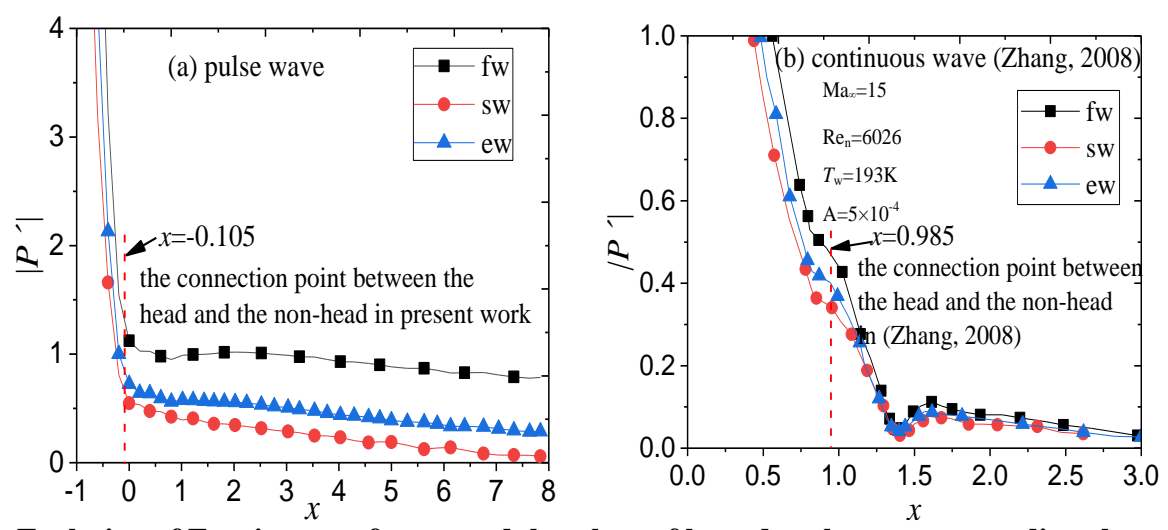

Fig. 10. Evolution of Fourier transforms module values of boundary layer pressure disturbance along the streamwise for freestream pulse waves (a) and continuous waves (Zhang et al. 2008) (b).

$\left(f_{2}-f_{6}\right)$ along the streamwise $\left(f_{\mathrm{n}}, \mathrm{n}=1,2,3,4,5,6\right)$ are shown in Fig. 11. Meanwhile, the developments of the $f_{1}$ mode, $f_{2}$ mode and $f_{3}$ mode under freestream continuous fast acoustic wave obtained by Zhang et al. (2008) are also shown in Fig.11(a)-(c).

From Fig.11, it is obtained that in the head area of the blunt cone $(s<\pi / 2-\theta=1.4312)$, the fundamental and harmonic frequencies $\left(f_{1}-f_{6}\right)$ have a significant attenuation trend. Among the three cases, behind the head area $(s>\pi / 2-\theta=1.4312)$, the amplitudes of $f_{1}$ and $f_{2}$ mode (the fundamental and second harmonic mode) in the boundary layer have no significant change, and remain basically stable. For the third-order harmonic mode, the amplitude increases continuously after a significant attenuation in the head area. For the fourth and sixth order harmonic mode, their amplitudes increase slightly in the $2<s<4$ area; when $s>4$, their amplitudes fall into decay again. For the fifth-order harmonic mode, their amplitudes increase significantly in the $2<s<5$ area, this increase once makes the amplitudes of $f_{5}$ in the $2<s<5$ area larger than that in the head area; when $s>5$, the amplitudes of $f_{5}$ falls into decay again. It can be seen that the type of pulse disturbance wave has a great influence on the evolution of fundamental and harmonic frequency modes. In the view of area, the type of freestream pulse waves has a significant effect on the disturbance modes both in the head area and non-head area. It should be pointed out that the boundary layer disturbance modes in the head area, especially the area near $s=0$ are mainly the initial waves induced by the interaction of external pulse wave with boundary layer and bow shock wave. Meanwhile, as mentioned above, the induced waves will interact with boundary layer again, induce new disturbance waves, and so forth; the boundary layer disturbance modes will change radically. This process is called the evolution of boundary layer disturbance modes. That is, the type of pulse disturbance wave has a great influence on the production and evolution of boundary layer disturbance wave.

At the same time, from the continuous fast acoustic wave simulations of Zhang et al. (2008) in Fig.11, there is an obvious attenuation trend for the amplitude of $f_{1}$ and $f_{2}$ modes in the head area. However, there is a small increase in the connection area (approximately $1.5<s<3$ ) between the head and the non-head, which remain stable or decreased slowly in the later area. For the third-order harmonic mode, under the case of pulse wave, the disturbance amplitude decreases in the head area, and increases continuously after the connection area between the head and the non-head $(s>1.5)$. However, under the case of continuous fast acoustic wave, the disturbance amplitude decreases in the head area, and increases significantly in the area of $1.3<s<1.7$, and then decreases after $s>1.7$. Based on the above analysis, it is obtained that, both for the case of freestream pulse wave and freestream continuous wave, the increase of disturbance amplitude or the decrease of attenuation rate appear in the connection area between the head and nonhead. It is considered that this phenomenon result from flow recompression, and the recompression effect induced by the configuration of blunt cone in the case of pulse wave is significantly different from that in the case of continuous wave.

Figure 12 shows the evolution trend of the amplitude growth rates of the fundamental mode $\left(f_{1}=0.25\right)$ and harmonic modes $\left(f_{2}-f_{6}\right)$ along the streamwise under different freestream pulse disturbance waves. From Fig.12, it can be seen that the type of pulse disturbance wave has a great influence on the evolution of boundary layer disturbance wave. Among the three kinds of freestream pulse disturbance waves, in the blunt cone head area $(s<1.4312)$, the amplitude growth rates of $f_{1}$ mode and harmonic modes of each order decrease continuously at first and then increase after the turning point near $s=1$. Except for the area near the turning point $s=1$, the sequence of the amplitude growth rate of $f_{1}, f_{2}, f_{3}, f_{4}$ and $f_{6}$ in the head area of blunt cone is Case2, Case 3 and Case1. However, in the vicinity of turning point $s=1$, the sequence of the amplitude growth rate of $f_{1}, f_{2}, f_{3}, f_{4}$ and $f_{6}$ is Case 3 , Case 2 and Case1. For the fifth harmonic mode in the head area, when $s<1.0$, the growth rate of $f_{5}$ is the largest in Case2, followed by Case 3 and Case1; when $1.0<s<1.4312$, Case 1 is the largest followed by Case 3, and Case2 is the smallest. In the non- 

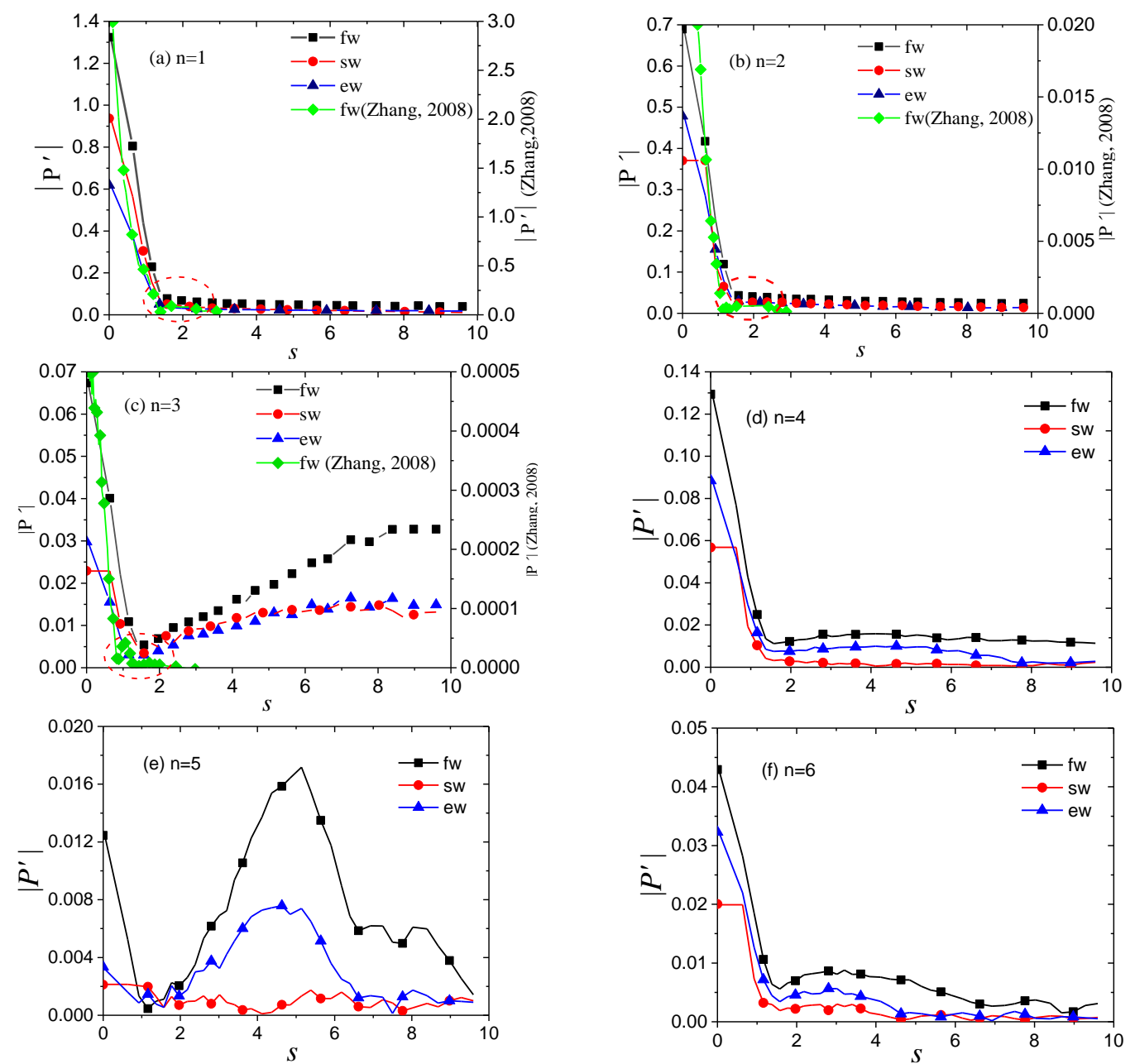

Fig. 11. Development of boundary layer pressure disturbance mode along the streamwise for different freestream disturbance waves (Zhang et al. 2008). (a) the development for $f_{l}$ mode. (b) the development for $f_{2}$ mode. (c) the development for $f_{3}$ mode. (d) the development for $f_{1}$ mode. (e) the development for $f_{5}$ mode. (f) the development for $f_{6}$ mode.

head area, the amplitude growth rates of the disturbance modes of each order under the three freestream pulse waves experience a short growth, and then the amplitude growth rates of different disturbance modes under different cases show different evolution trends along the streamwise. In general, their growth rates or attenuation rates decrease significantly in magnitude.

Figure 13 shows the average growth rates of different disturbance modes $\left(f_{1}-f_{6}\right)$ in the head area, connection area and non-head area under different pulse waves. It can be seen from Fig.13 that, 1) in the head area, the average growth rate of $f_{1}-f_{6}$ modes is less than 0 under the three freestream pulse waves, which indicates that in the head boundary layer, $f_{1}-f_{6}$ modes under the three freestream pulse waves show a negative growth trend. Moreover, among the three kinds of freestream pulse waves, when $1 \leq n \leq 5$, the average growth rate of the boundary layer disturbance modes increases with the increase of the order of the mode. At the same time, it also is obtained that the growth rate of $f_{6}$ is less than that of $f_{5}$. In the head area, the average growth rate of $f_{1}-f_{6}$ modes is the highest in the case of pulse slow acoustic wave, the case of pulse entropy wave takes second place. 2) In the connection area between head and non-head, the variation tendency of the average growth rate of the disturbance modes of each order $\left(f_{1}-f_{6}\right)$ is similar under the three kinds of freestream pulse disturbance waves. That is, relative to that in the head area, the average negative growth rate of the fundamental and second harmonic modes decreases, and the average growth rate of $f_{3}-f_{6}$ changes from negative growth in the head area to positive growth in the connection area (except for $f_{3}$ mode under Case2). In the connection area, the flowfield recompression effect caused by the computational model configuration plays a key role in the evolution of each order modes under the three kinds of pulse waves. It can also be seen that in the connection area, from the point of view of the absolute value of the average growth rate of each order disturbance mode, the case of fast acoustic wave case is the largest, the case of entropy wave is the second, and the case of slow acoustic is the smallest. From the data in the head area and the 

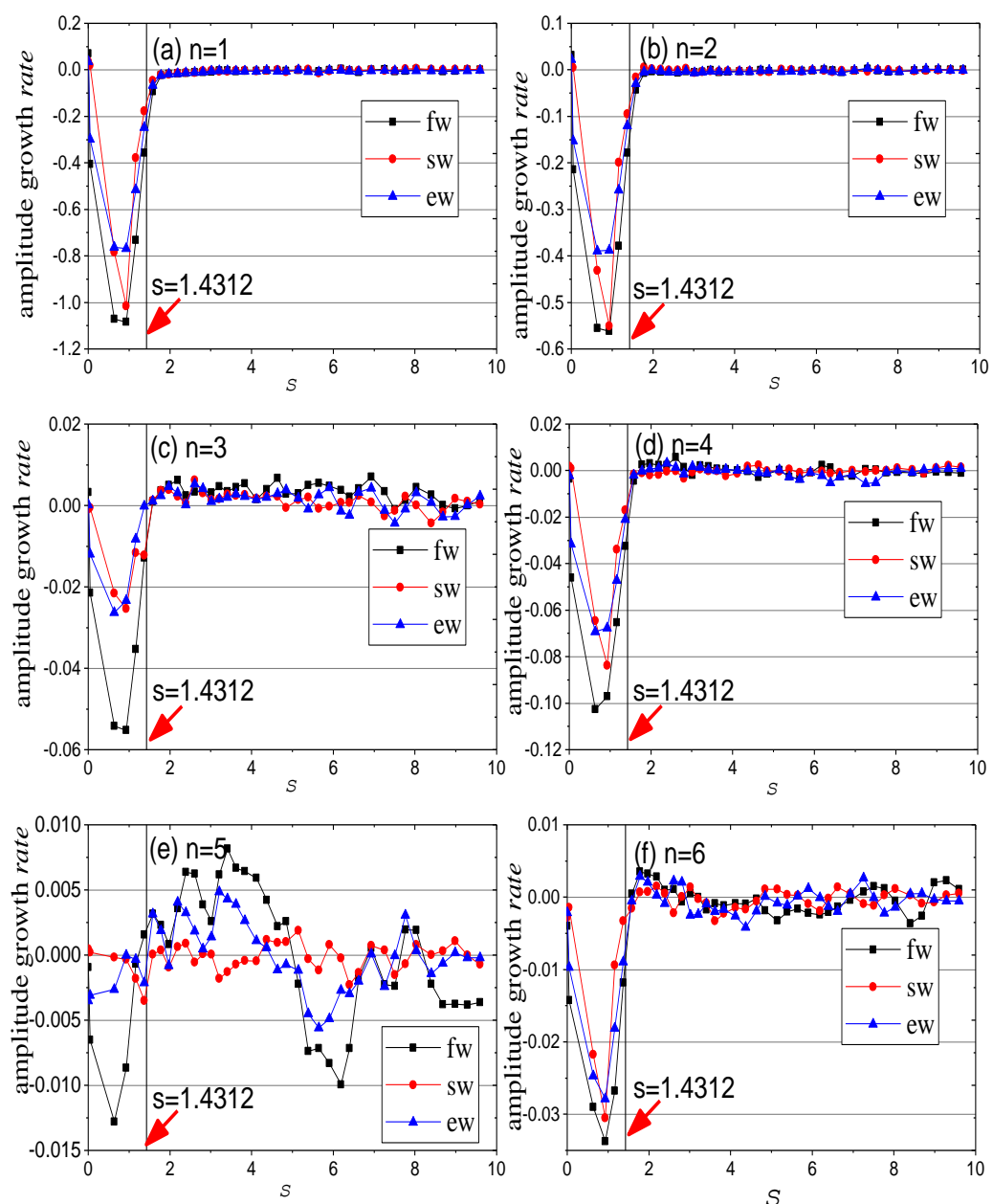

Fig. 12 Evolution trend of the amplitude growth rates of different disturbance modes along the streamwise under different freestream pulse waves. (a) the growth rate for $f_{l}$ mode. (b) the growth rate for $f_{2}$ mode. (c) the growth rate for $f_{3}$ mode. (d) the growth rate for $f_{1}$ mode. (e) the growth rate for $f_{5}$ mode. (f) the growth rate for $f_{6}$ mode.
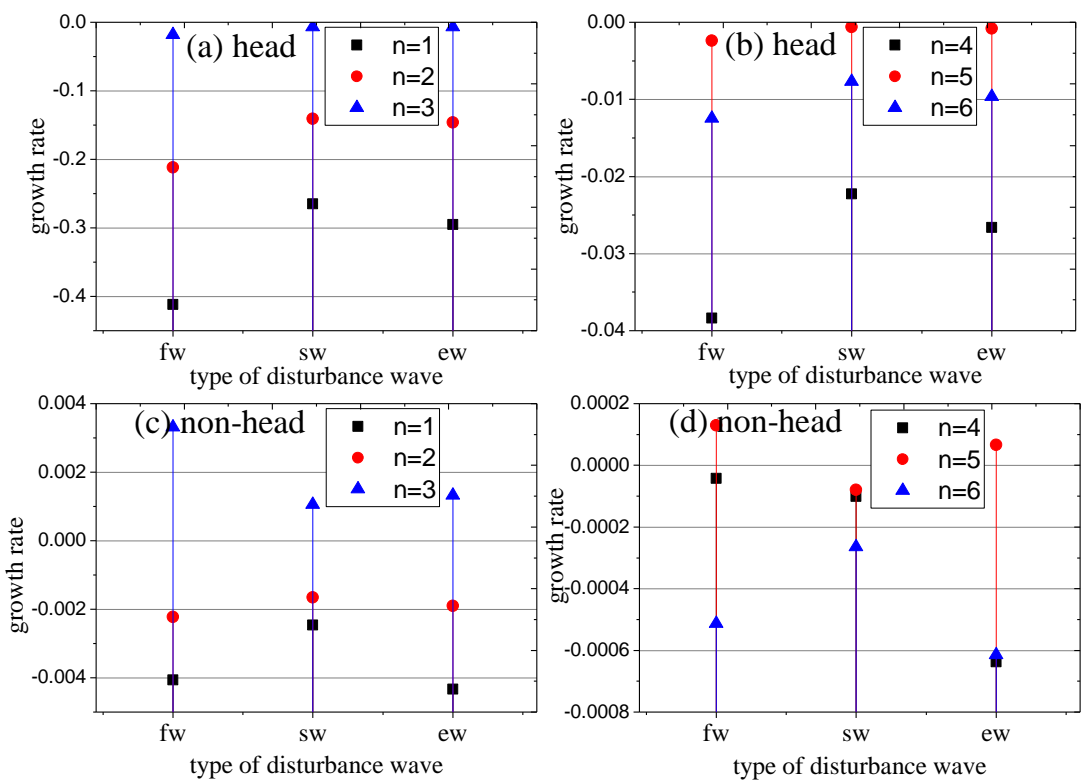

Fig. 13. Comparison of the average growth rates of different disturbance modes $\left(f_{1}-f_{6}\right)$ in the head area, connection area and non-head area under different pulse waves. (a) $f_{1}, f_{2}$ and $f_{3}$ modes in the head boundary layer. (b) $f_{4}, f_{5}$ and $f_{6}$ modes in the head boundary layer. (c) $f_{1}, f_{2}$ and $f_{3}$ modes in the nonhead boundary layer. (d) $f_{4}, f_{5}$ and $f_{6}$ modes in the non-head boundary layer. 
connection area, it is concluded that under the action of pulse slow acoustic waves, the disturbance wave evolution of each order mode in the boundary layer along the streamwise is relatively stable, followed by entropy wave, and the case of fast acoustic wave is the most active. 3) In the non-head area, for the absolute value of the average growth rate of $f_{2}, f_{3}$ and $f_{6}$, the case of fast acoustic wave is the largest, the case of entropy wave is the second, and the case of slow acoustic wave is the smallest; for the absolute value of the average growth rate of $f_{1}, f_{4}$ and $f_{5}$, the case of entropy acoustic wave is the largest, followed by the cases of fast and slow acoustic wave.

\section{CONClusion}

The DNS of hypersonic blunt cone flow with finite amplitude pulse waves in the freestream is carried out. The response characteristics of hypersonic boundary layer among different types of pulse disturbance are studied. The following conclusions are drawn.

1) The mechanism of the interaction of different freestream pulse disturbance waves with strong shear flow structure and thermal state of boundary layers is significantly different. The influence of the mainstream disturbance wave generated by the pulse fast acoustic wave on the boundary layer thermodynamic characteristics is greater than that of the pulse entropy wave and pulse slow acoustic wave. The reflected wave generated by the pulse slow acoustic wave has greater influence on the boundary layer thermodynamic characteristics than that of the cases of pulse fast and slow acoustic wave.

2) The type of pulse disturbance wave has a great influence on the production and mode distribution of boundary layer disturbance wave. In the process of disturbance wave evolution, there exists a disturbance mode group's migration phenomenon in the three cases of pulse waves. The main mode groups attenuation phenomenon which narrows the frequency band of the disturbance waves exists in all of the three cases, and the fastest attenuation occurs in the condition of pulse slow acoustic wave, followed by the condition of pulse entropy wave and the slowest in the condition of pulse fast acoustic wave.

3) In the head area, except for the area near the turning point, the order of the growth rate of each order modes except the fifth order harmonic mode is the condition of slow acoustic, entropy and fast acoustic wave. However, near the turning point, the sequence of the growth rate of the disturbance modes except the fifth harmonic mode is the case of entropy wave, slow acoustic wave and fast acoustic wave. In non-head area, the growth rate of different disturbance modes presents different variation trend under the three cases of freestream pulse wave, and their growth rate or attenuation rate decreases significantly. Under the action of pulse slow acoustic waves, the disturbance wave evolution of each order mode in the boundary layer along the streamwise is relatively stable, followed by entropy wave, and the case of fast acoustic wave is the most active.

\section{ACKNOWLEDGEMENTS}

This paper is funded by the equipment pre-research common technology project of the 13th five years plan (Grant No. 41402010201).

\section{REFERENCES}

Chen, Z., Y. Zhao and R. Huang (2018). Reducedorder modeling of unsteady hypersonic aerodynamics in multi-dimensional parametric space. Journal of Applied Fluid Mechanics 11(4), 1033-1045.

De, T. N. (2013). Receptivity and transition to turbulence of supersonic boundary layers with surface roughness. $\mathrm{Ph}$. D. thesis, University of Southampton, Southampton, United Kingdom.

Duan, L., P. Martin and I. Beekman (2010). Direct numerical simulation of hypersonic turbulent boundary layers with varying freestream Mach number. In Proceedings of 48th AIAA Aerospace Sciences Meeting Including the New Horizons Forum and Aerospace Exposition, Orlando, America.

Egorov, I. V., A. V. Fedorov and V. G. Soudakov (2008). Receptivity of a hypersonic boundary layer over a flat plate with a porous coating. Journal of Fluid Mechanics 601, 165-187.

Egorov, I. V. and A. V. Novikov (2016). Direct numerical simulation of laminar-turbulent flow over a flat plate at hypersonic flow speeds. Computational Mathematics and Mathematical Physics 56(6), 1048-1064.

Finley, P. J (1966). The flow of a Jet from a body opposing a supersonic free stream. Journal of Fluid Mechanics 26, 337-368.

Fujii, K (2015). Experiment of the two-dimensional roughness effect on hypersonic boundary-layer transition. Journal of Spacecraft \& Rockets 43(4), 731-738.

Gao, J., J. S. Luo and X. S. Wu (2015). Receptivity of hypersonic boundary layer due to fast-slow acoustics interaction. Acta Mechanica Sinica 31(6), 899-909.

Hayashi, K. and S. Aso (2004). A study on reduction of aerodynamic heating by opposing jet in supersonic flow. Journal of the Japan Society for Aeronautical and Space Sciences $52,38-44$

Huang, Y., X. Zhong (2014). Numerical study of hypersonic boundary-layer receptivity with freestream hotspot perturbations. AIAA J. 52(12), 2652-2672.

Kara, K., P. Balakumar and O. A. Kandil (2011). 
Effects of nose bluntness on hypersonic boundary-layer receptivity and stability over cones. AIAA J. 49(12), 2593-2606.

Li, X. L., D. X. Fu and Y. W. Ma (2008). DNS of compressible turbulent boundary layer around a sharp cone. Science in China Series $G$ (Physics, Mechanics and Astronomy) 51(6), 699-714.

Li, X., D. Fu and Y. Ma (2010). Direct numerical simulation of hypersonic boundary layer transition over a blunt cone with a small angle of attack. Physics of Fluids 22(2), 025105.

Liu, X. D., S. Osher and T. Chan (1994). Weighted essentially non-oscillatory schemes. Journal of Computational Physics 115, 200-212.

Lobb, R. K. (1964). The high temperature aspects of hypersonic flow-Experimental measurement of shock detachment distance on spheres fired in air at hypervelocities. Pergamon Press, Oxford, UK.

Prakash, A., N. Parsons, X. Wang and X. Zhong (2011). High-order shock-fitting methods for direct numerical simulation of hypersonic flow with chemical and thermal nonequilibrium. Journal of Computational Physics 230(23), 8474-8507.

Qin, F. and X. Wu (2016). Response and receptivity of the hypersonic boundary layer past a wedge to free-stream acoustic, vortical and entropy disturbances. Journal of Fluid Mechanics 797, 874-915.

Shiplyuk, A., S. Lukashevich, D. Bountin, A. Maslov and H. Knaus (2013). Experiments on hypersonic boundary layer transition on blunt cones with acoustic-absorption coating. Progress in Flight Physics 3, 295-304.

Sivasubramanian, J. and H. F. Fasel (2015). Direct numerical simulation of transition in a sharp cone boundary layer at Mach 6: fundamental breakdown. Journal of Fluid Mechanics 768, 175-218.

Tang, X., H. Lv, X. Meng, Z. Wang and Q. Lv (2014). Stability characteristic of hypersonic flow over a blunt wedge under freestream pulse wave. Central European Journal of Physics 12(1),17-31.

Tang, X., X. Zhu, T. Hui, W. Yu, F. Yang and C. Cao (2017). Receptivity characteristics of a hypersonic boundary layer under freestream slow acoustic wave with different amplitudes. European Physical Journal Applied Physics 79(3), 31101.

Venukumar, B., G. Jagadeesh and P. J. K. Reddy (2006). Counter flow drag reduction by supersonic jet for a blunt body in hypersonic flow. Physics of Fluids 18(11), 571-576.

Voland, R. T., L. D. Huebner and C. R. Mcclinton (2005). X-43A Hypersonic vehicle technology development. Acta Astronautica 59(1),181191.

Wan, B. B., J. S. Luo and C. H. Su (2018). Response of a hypersonic blunt cone boundary layer to slow acoustic waves with assessment of various routes of receptivity. Applied Mathematics and Mechanics 39(11),1643-1660

Wang, X. and X. Zhong (2006). Numerical simulation of hypersonic boundary-layer receptivity to three-dimensional wall perturbations. AIAA Paper AIAA2006-3225.

Wang, Z., M. Shi, X. Tang, H. Lv and L. Xu (2018). Effect of a roughness element on the receptivity of a hypersonic boundary layer over a blunt cone due to pulse entropy disturbance with a Single Frequency. Entropy 20(6), 404.

Wang, Z., X. Tang and H. Lv (2014). Response of a hypersonic boundary layer to freestream pulse acoustic disturbance. The Scientific World Journal 2014, 748504.

Zhang, C., Y. Zhu, X. Chen, H. Yuan, J. Wu, S. Chen, C. Lee and M. Gad-el-Hak (2015). Transition in hypersonic boundary layers. AIP Advances 5(10), 79-147.

Zhang, Y., D. Fu, Y. Ma, and X. Li (2008). Receptivity to free-stream disturbance waves for hypersonic flow over a blunt cone. Science in China, Series G: Physics, Mechanics and Astronomy 51(11), 1682-1690.

Zhao, R., C. Y. Wen, X. D. Tian, T. H. Long and W. Yuan (2018). Numerical simulation of local wall heating and cooling effect on the stability of a hypersonic boundary layer. International Journal of Heat \& Mass Transfer 121, 986998.

Zhao, Y. F., W. Liu, D. D. Gang, S. H. Yi and X. G. Deng (2015). Study of surface roughness induced supersonic boundary layer transition. Journal of Astronautics 36(6), 739-746.

Zhong, X. L (2001). Leading-edge receptivity to free-stream disturbance waves for hypersonic flow over a parabola. Journal of Fluid Mechanics 441, 315-367.

Zhong, X. L and J. Lei (2011). Numerical simulation of nose bluntness effects on hypersonic boundary layer receptivity to freestream disturbances. AIAA Paper AIAA2011-3079. 